\title{
Effect of surfactant for magnetic properties of iron oxide nanoparticles.
}

\author{
S. Haracz ${ }^{1}$, M. Grzeszkowiak ${ }^{2}$, M. Hilgendorff ${ }^{3}$, J. D. Rybka ${ }^{1}$, M. Giersig ${ }^{1,3}$ \\ 1) Faculty of Chemistry, Adam Mickiewicz University, Umultowska 89B, 61-614 Poznań, Poland \\ 2) The NanoBioMedical Centre, Adam Mickiewicz University, Umultowska 85, 61-614 Poznań , Poland \\ 3) Freie Universität Berlin, FachbereichPhysik, Arnimalle 14, 14195 Berlin, Germany
}

Abstract: For a different medical applications nanoparticles (NPs) with well-defined magnetic properties have to be used. Coating ligand can change the magnetic moment on the surface of nanostructures and therefore the magnetic behavior of the system. Here we investigated magnetic NPs in a size of $13 \mathrm{~nm}$ conjugated with four different kind of surfactants. The surface anisotropy and the magnetic moment of the system was changed due to the present of the surfactant on the surface of iron oxide NPs.

\section{Keywords: nanoparticles, magnetic moment, anisotropy of the surface, superparamagnetism}

\section{Introduction}

Magnetic nanoparticles (NPs) are commonly researched because of possibilities to use them in medicine as a contrast agent, for cell separation or drug delivery. Important parameter for magnetic behavior are kind of synthesis, shape, size and organic ligand bind to the surface.

A number of groups report results which have shown that ligand have influence on susceptibility, magnetization and coercivity $[1,2]$.

Here we present four different samples in the same average size of $13 \mathrm{~nm}$, but with different substances bonded to the surface. We measured dynamic and static magnetic properties. All samples are in superparamagnetic state in room temperature where the magnetic moment can fluctuate between two anisotropy easy axis. 


\section{Synthesis}

\subsection{Synthesis of oleic acid (OA) coated iron oxide NPs}

Iron oxide NPs were prepared by thermal decomposition in a organic solution using Sun's method [3,4]. The NPs were dispersed in chloroform and transferred to physiological solution or water using three different techniques:

\subsection{Coating of iron oxide NPs with CTAB[5]}

To the solution of NPs in chloroform $0.045 \mathrm{~g}$ cetyltrimethylammonium bromide (CTAB) was added and stirred. For a better separation distillation process was used in order to remove chloroform. System was redispersed in water and centifugated several times.

1.3 Coating of iron oxide NPs with mPAA -mPAA - PEG

\section{Polimer synthesis}

Modify polyacrylic acid (mPAA- $1 \mathrm{~g}$ ) was prepared after Bawendi group[6]. $1 \mathrm{~g}$ Polyacrylic acid was dissolved in $10 \mathrm{ml}$ DMF(dimethyloformamide). $0.72 \mathrm{~g} \mathrm{~N}$-octyl amine was added into solution. The reaction mixture was stirred for 2 hours before 1,06 g 1-ethyl-3-(3-dimethylaminopropyl)carbodiimide(EDC) was added. In the next step mixture was stirred at room temperature for 20 hours. DMF was then removed under reduce pressure and $2 \mathrm{ml}$ of water with $1 \mathrm{~g}$ of tetramethylammonium hydroxide was added and stirred for $2 \mathrm{~h}$. After stirring $4 \mathrm{ml} 1.3 \mathrm{M}$ hydrochloric acid was added in order to re-precipitate the MPAA and the supernatant was then removed. The purified mPAA was then dissolved and kept in ethylacetate.

\section{Coating process of iron oxide nanoparticles}

$5 \mathrm{mg}$ of magnetic NPs was mixed with mPAA in chloroform for $12 \mathrm{~h}$. Chloroform was slowly reduced by pressure and dropwise addition of PBS (Phosphate buffer saline) during the sonication. 


\section{Modified mPAA - mPAA - PEG}

mPAA coated magnetic NPs in PBS were mixed with $0,003 \mathrm{~g}$ EDC for $2 \mathrm{~h}$ and $10 \mu \mathrm{L}$ O-(2aminopropyl)O'(2-methoxyethyl)polypropylene ethylene glycol was added. Solution was stirred for $12 \mathrm{~h}$.

Magnetic NPs in PBS were modified by amino group using EDC/NHS[7] technique by O-(2aminopropyl)O'(2-methoxyethyl)polypropylene ethylene glycol.

\subsection{Redispersion in water with Sodium hyaluronan HA}

$0,1 \mathrm{mg}$ of magnetic NPs were redispersed in $5 \mathrm{ml}$ chloroform and $13 \mathrm{ml}$ water with $0,01 \mathrm{~g}$ sodium hyaluronan. Then the solution was mixed for $20 \mathrm{~h}$. Afterwards chloroform was removed by reducing pressure and nanostructures were redispersed in water. NPs were then cleaned by centrifugation (in order to remove the exceed of hyaluronan) and finally the supernatant was exchanged by water.

\section{Discussion}

\subsection{TEM and Raman spectroscopy characterization of the coated iron oxide} NPs

A morphology of iron oxide nanoparticles was measured using Transmission Electron Microscopy (TEM) a $120 \mathrm{keV}$ JOEL JEM - 1400. All samples are spherical and monodisperse. The average size of our four systems was $13 \mathrm{~nm}$, see Figure 1. For later discussion about magnetic anisotropy, it is important that nanostructures had almost the same size, see Table 1.

Figure 1. TEM image of iron oxide nanoparticles coated by a)oleic acid b)CTAB c) MPAA-PEG d)HA.

To ensure that ligand exchange process was correct we measured Raman spectra of our samples which have been shown on Figure 2. Because of high absorption of our systems, laser with wavelength of $785 \mathrm{~nm}$ was used for all samples.

Figure 2. Raman spectra of iron oxide nanoparticles coated by a) CTAB b)mPAA c) $H A$. 
Iron oxide coated by CTAB present peaks from alkyl groups $\left(1061 \mathrm{~cm}^{-1}, 1288 \mathrm{~cm}^{-1}\right.$, from $2735 \mathrm{~cm}^{-1}$ to $\left.2880 \mathrm{~cm}^{-1}\right)$. Moreover bands from nitrogen-carbon bonds $\left(952 \mathrm{~cm}^{-}\right.$ ${ }^{1}, 1526 \mathrm{~cm}^{-1}$ ) were observed. The typical peaks at $1153 \mathrm{~cm}^{-1}$, which correlates with vibration of $\mathrm{C}-\mathrm{C}$ chain were also visible.

For sample coated by mPAA-PEG some bonds from our ligand on the surface were observed. Following peaks were observed: alkyl groups $\left(1057 \mathrm{~cm}^{-1}, 1091 \mathrm{~cm}^{-1}\right)$, hydroxyl $\left(1387 \mathrm{~cm}^{-1}\right)$ and oscillation comes from $\mathrm{CNH}$ group $\left(1556 \mathrm{~cm}^{-1}\right)$ [8].

NPs stabilized by HA revealed typical peaks for iron oxide structures $\left(235 \mathrm{~cm}^{-1}\right)$ and some peaks which are related to the structure of sodium hyaluronan. Furthermore oscillation of carbon - oxide $\left(445 \mathrm{~cm}^{-1}, 825 \mathrm{~cm}^{-1}, 1112 \mathrm{~cm}^{-1}, 1178 \mathrm{~cm}^{-1}\right)$, hydroxyl groups $\left(1300 \mathrm{~cm}^{-1}\right)$ and double bond $\mathrm{C}=\mathrm{O}\left(1606 \mathrm{~cm}^{-1}\right)$ was observed.

\subsection{Magnetic properties of coated iron oxide NPs}

The fluctuating magnetic moment between two easy axis[9], which is isolated by energy barrier could be estimated using Zero-Filed Cooling (ZFC) and Field- Cooling (FC) techniques. In ZFC samples were cooled without magnetic field, and after that heated in very week magnetic field (100 Oe). In FC technique samples were cooled and heated in the same value of the field, see Figure 3.

Figure 3. Zero-field-cooling and field- cooling for iron oxide nanoparticles stabilized by a)oleic acid and CTAB b) MPAA-PEG and HA.

The blocking temperatures for samples coated by oleic acid, CTAB, mPAA - PEG and $\mathrm{HA}$ were estimated and are equal $74 \mathrm{~K}, 70 \mathrm{~K}, 72 \mathrm{~K}$ and $71 \mathrm{~K}$ respectively. The blocking temperatures for all ligand coated iron oxide particles were similar.

The dynamic properties of our nanostructures were analyzed with susceptibility in-phase (real part) and out-of-phase (imaginary part). Furthermore, AC measurements give information about interactions between NPs, see Figure 4. AC measurements versus temperature were obtain for eight different frequencies in a range from $10 \mathrm{~Hz}$ to $1488 \mathrm{~Hz}$.

Figure 4. Dependence of in-phase suspectibility for a) oleic acid b)CTAB c)mPAAPEG d)HA. 
Typical behavior of superaparamagnetic NPs is shift of the blocking temperature with increased frequency. The value of shifting is given by[10,11] :

$$
\Phi=\frac{\Delta T_{B}}{T_{B} \Delta \log _{10}(f)}
$$

This deviation is the simplest way to describe the quality and quantivity of interactions in superaparamagnetic systems. When the value of this parameter ranged from 0,005 to 0,01 then the system bahave as a spin glass, for the value between 0,01 0,013 the system is superparamagnetic with weak interactions (non-interacting nanosystems) $[12,13,14]$.

For our samples this deviations for different ligands on surface nanoparticles gives values between $0,012-0,031$, see Table 2. For NPs stabilized by mPAA PEG and HA parameter $\phi$ is between $0,01-0,013$, so we can conclude that interactions between NPs clusters are weak. Therefore samples can be treated as non-interacting nanosystems. For samples coated by CTAB and oleic acid the value of this parameter is higher, so in this nanostructures the interactions between nanoclusters are stronger.

The energy barrier between two easy axis could be estimate by Zero-Field Cooling and Field -Cooling measurement (ZFC, FC). Time necessary for changing the magnetic moment is correlated with time relaxation:

$$
\tau(T)=\tau_{0} \exp \left(E_{A} / k_{B} T\right)
$$

where $E_{A}$ is the anisotropy energy barrier. For single domain NPs the height of energy barrier corresponds with thermal energy. Below temperature which is called blocking temperature, the thermal energy is not able to break interactions between NPs and the system is in the "frozen" state. Above blocking temperature nanoparticles starts to be in the superparamagnetic state and the susceptibility became independent from frequency. 
Dynamic response of the superparamagnetic systems is correlated with relaxation time necessary for exchange direction of the magnetic moment between two easy axis[15,16,17]. Rotation along axis is correlated to the energy barrier which can be fixed from Arrhenius law (eq. 1). For sample coated by mPAA -PEG this value is the smallest one because the interactions in this system are weak. For NPs coated by oleic acid or CTAB energy barrier is higher because of strong interactions between nanoparticles, see Figure 5 and Table 2.

Figure 5. Logarithm time relaxation function of temperature a) oleic acid b) CTAB c) $m P A A-P E G$ d)HA.

All parameters which describes magnetic properties for ours systems are completely different because of modified surface of nanoparticles with organic ligands. Therefore magnetic moment of surfaces is a key for solution of this behavior. We can estimate effective anisotropy using TEM images and solving the equation:

$K_{e f f}=\frac{6 \cdot E_{a}}{\pi \cdot D^{3}{ }_{T E M}}(4)$

where $E_{a}$ is energy barrier from Table 2. In the simplest approximation $E_{a}$ equals a sum of anisotropy of volume and surface (eq. 3). Our results are summarized in Table 2.

For all samples we could see the same magnitude of effective anisotropy. But for the NPs stabilized by mPAA- PEG and HA the value of energy barrier are bigger than for other two ligands. From magnetic measurments we concluded that nanoparticles coated by mPAA -PEG and HA are superparamagnetic without interactions. For all samples we calculated the effective aniosotropy. As we observed for this two ligands the value of this physical parameter is equal. But for nanostructures coated by CTAB and oleic acid the effective anisotropy is lower, see Table 2.

For different organic ligand on surface we change the anisotropy of the surface. For simplest approximation we could calculate the effective anisotropy for systems by[18,19]:

$K_{\text {eff }}=K_{V}+(6 \Phi / D) K_{S}$ 
where $K_{V}$ is the bulk anisotropy energy per unit volume, $K_{S}$ is the surface density of anisotropy energy and $6 \Phi / D$ the surface to volume ratio. The change of effective anisotropy after coating process is shown. For NPs bonded to mPAA - PEG and HA the values of this parameter were increased and decreased for the nanosystem coated by CTAB.

For all four samples we measured hysteresis loop for five different temperatures between 5-270 K, see figure 6 . In temperatures $5 \mathrm{~K}$ all samples have typical hysteresis loop for ferromagnetic material. For temperatures $50 \mathrm{~K}$ the value of coercivity for all samples decreased, which is typical behaviuor of superparamagnetic state. Moreover, the saturation magnetization decreased with the increasing temperature, because of the thermal movements. All values of coercivity and saturation magnetization changed with temperatures are collected in Table 3.

Figure 6. Hysteresis loops a) $T=5 \mathrm{~K}$ b) $T=270 \mathrm{~K}$.

\section{Conclusions}

We presented here four sample with the same average size around $13 \mathrm{~nm}$ with different organic ligands on the surface. We could observed that the type of binding to the surface of the NPs have influence on magnetic properties such as susceptibility, barrier energy and effective anisotropy. For the medical applications we would like to obtain NPs with high saturation magnetization at room temperatures. Because we want to have NPs well-separated (with small interaction), we can conclude from measurements that NPs coated by mPAA- PEG and HA are the most stabilized. Organic ligand is changing the magnetic moment on the surface of NPs and therefore using different stabilizer manipulation of magnetic moment and magnetic properties of the nanosystem is possible.

\section{Acknowledgements}

This work was supported by UMO-2012/06/A/ST4/00373 grant from National Science Centre (Poland). 


\section{References:}

[1] M. Filippousi , M. Angelakeris, M. Katsikini , E. Paloura , I. Efthimiopoulos , Y. Wang, D. Zamboulis, G. Van Tendeloo, Surfactant Effects on the Structural and Magnetic Properties of Iron Oxide Nanoparticles, J. Phys. Chem. C (2014),118, 16209-16217

[2] P. Soares , A.M. Alves , L.C. Pereira , J.T. Coutinho, I.M. Ferreira , C.M. Novo , J.P. Borges, Effects of surfactants on the magnetic properties of iron oxide colloids, J Colloid Interface Sci. (2014), 419, 46-51

[3] T. Hyeon, S. Seong Lee, J. Park, Y. Chung, and Hyon Bin Na, Synthesis of Highly Crystalline and Monodisperse Maghemite Nanocrystallites without a Size-Selection Process, J. Am. Chem. Soc.123 (2001), 123, 12798-12801.

[4] J. Park, K. An, Y. Hwang, J. Park, H. Noh, J.Kim, J. Park, N.Hwang and T. Hyeon, Ultra-large-scale syntheses of monodisperse nanocrystals,Nature Materials (2004), 3, 891-895.

[5] K. Khoshnevisan, M. Barkhi, D. Zare, D. Davoodi, M. Tabatabaei, Preparation and Characterization of CTAB-Coated Fe3O4 Nanoparticles, Synthesis and Reactivity in Inorganic, Metal-Organic, and Nano-Metal Chemistry (2012) ,42, 644648

[6] N. Insin, Surface Modifications of Iron Oxide Nanoparticles for Biological Applications PhD thesis, Massachusetts Institute of Technology, Boston (2011).

[7] Y. Kim, W. Kim, H. Yoo and S. Koo Shin, Bioconjugation of Hydroxylated Semiconductor Nanocrystals and Background-Free Biomolecule Detection, Bioconjugate Chem. (2012), 21, 1305-1311.

[8] C. Murli and Y. Song, Pressure-Induced Polymerization of Acrylic Acid: A Raman Spectroscopic Study, J Phys Chem B (2010), 114, 9744-9750.

[9] R. H. Kodama, , Magnetic nanoparticles, Journal of Magnetism and Magnetic Material (1999), 200, 359-372. 
[10] G.F. Goya, T.S. Berquó and F.C. Fonseca, Static and dynamic magnetic properties of spherical magnetite nanoparticles, Journal of Applied Physics (2003), 94, 3520-3528.

[11] A.C. Roca, D. Carmona, N. Miguel- Sancho, O. Bomat-Miguel, F. Balas, C. Piquer and J. Santamaria, Surface functionalization for tailoring the aggregation and magnetic behaviour of silica-coated iron oxide nanostructures, Nanotechnology (2012), 23 , 155603-155613.

[12] C.E. Botez, J. L. Morris, M. P. Eastman, Superspin relaxation in Fe3O4/hexane magnetic fluids: a dynamic susceptibility study, Chemical Physics (2012),403, 89-93.

[13] M. El-Hilo, K. O'Grady and R.W. Chartell, Susceptibility phenomena in a fine particle system: I. Concentration dependence of the peak, Journal of Magnetic Materials (1992),114, 295-306.

[14] J. L. Dormann, L. Bessais and D. Fiorani, A dynamic study of small interacting particles: superparamagnetic model and spin-glass laws, J. Phys. C: Solid State Phys. (1987), 21, 2015-2034.

[15] A. Zelenakova, V. Zelenak, J. Kovac, The magnetic behaviour of iron oxide nanoparticles prepared by self assembly array, Acta Electrotechnicaet Informatica (2010), 10, 39-42.

[16] J. L. Dormann, D. Fiorani, R. Cherkaoui, E. Tronc, F. Lucari, F. D'Orazio, L. Spinu, M. Nogues, H. Kachkachi, J.P. Jolivet, From pure superparamagnetism to glass collective state in gamma- $\mathrm{Fe} 2 \mathrm{O} 3$ nanoparticle assemblies, Journal of Magnetism and Magnetic Material (1999), 118, 23-27.

[17] P. Poddar, T. Telem - Shafir, T. Fried and G. Markovich, Dipolar interactions in two- and three-dimensional magnetic nanoparticle arrays, Physical Review B (2002), 66, 060403-1-060403-4.

[18] L.F. Gamarra , G.E.S. Brito, W.M. Pontuschka, E. Amaro, A.H.C. Parma, G.F. Goya, Biocompatible superparamagnetic iron oxide nanoparticles used for contrast agents: a structural and magnetic study, Journal of Magnetism and Magnetic Materials (2005), 289, 439-441. 
[19] A. Demortiere, P. Panissod, B. P. Pichon, G. Pourroy, D. Guillon, B. Donnio and S. Begin-Colin, Size-dependent properties of magnetic iron oxide nanocrystals, Nanoscale (2011), 3, 225-232. 


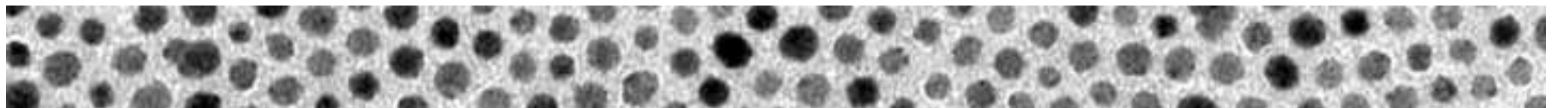

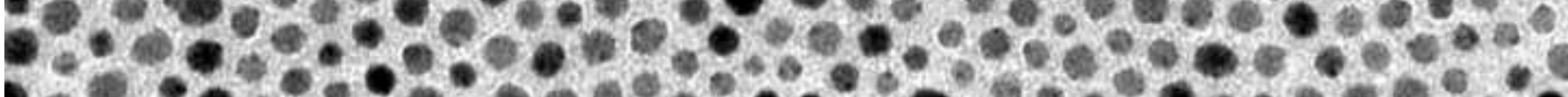

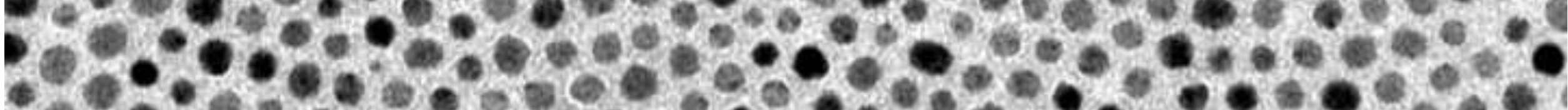

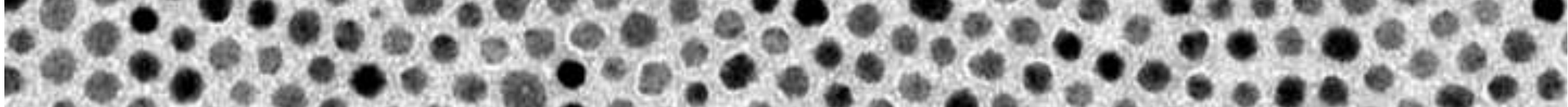
4 4.

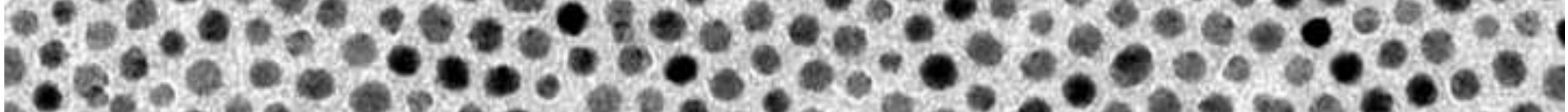

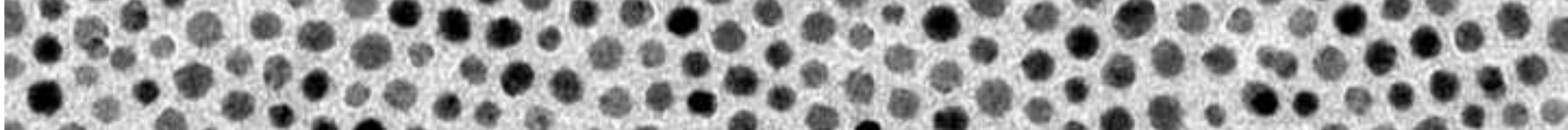

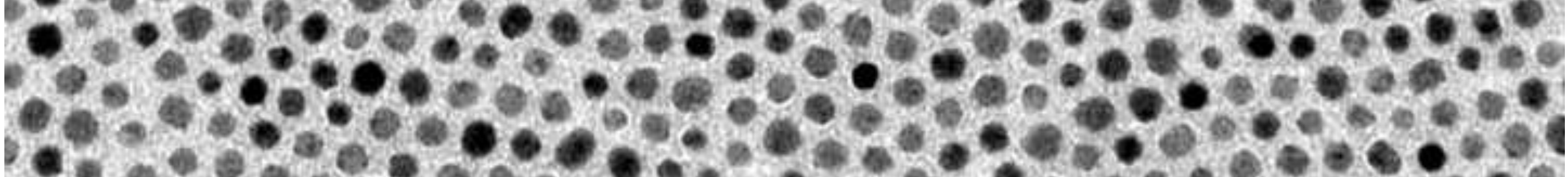

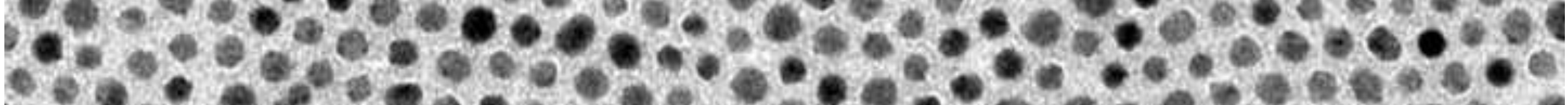

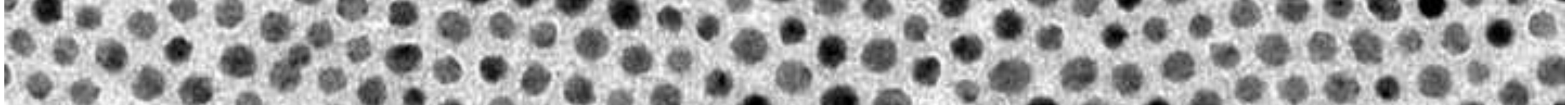

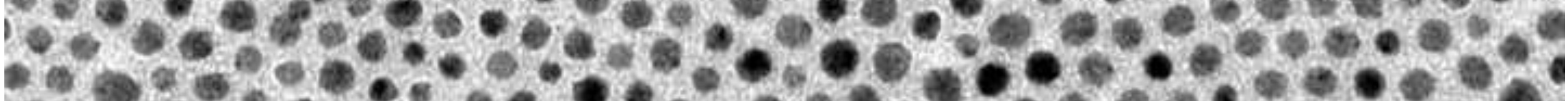

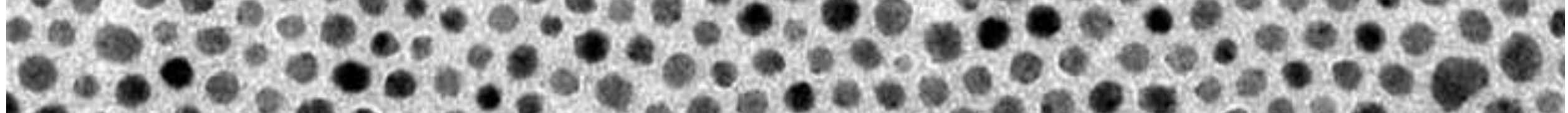

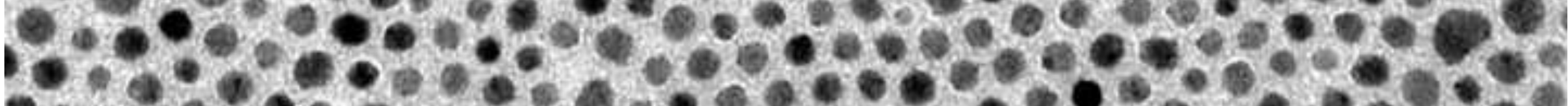

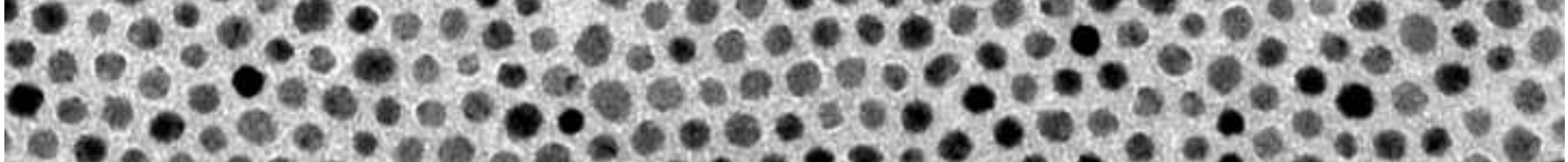

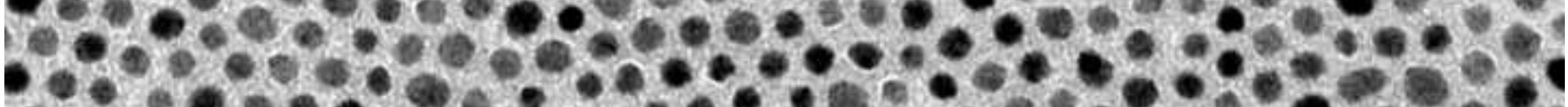

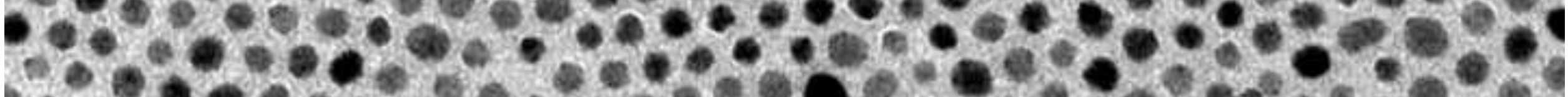

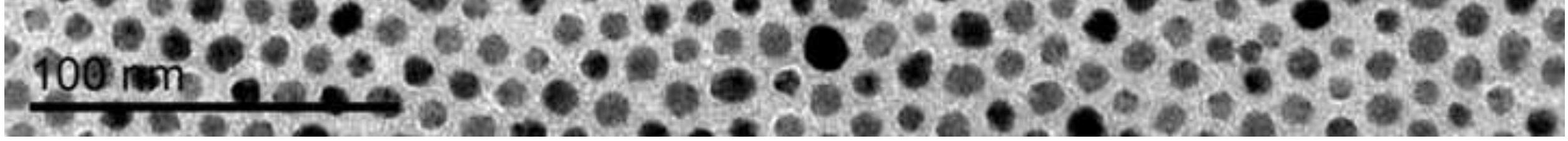
样 


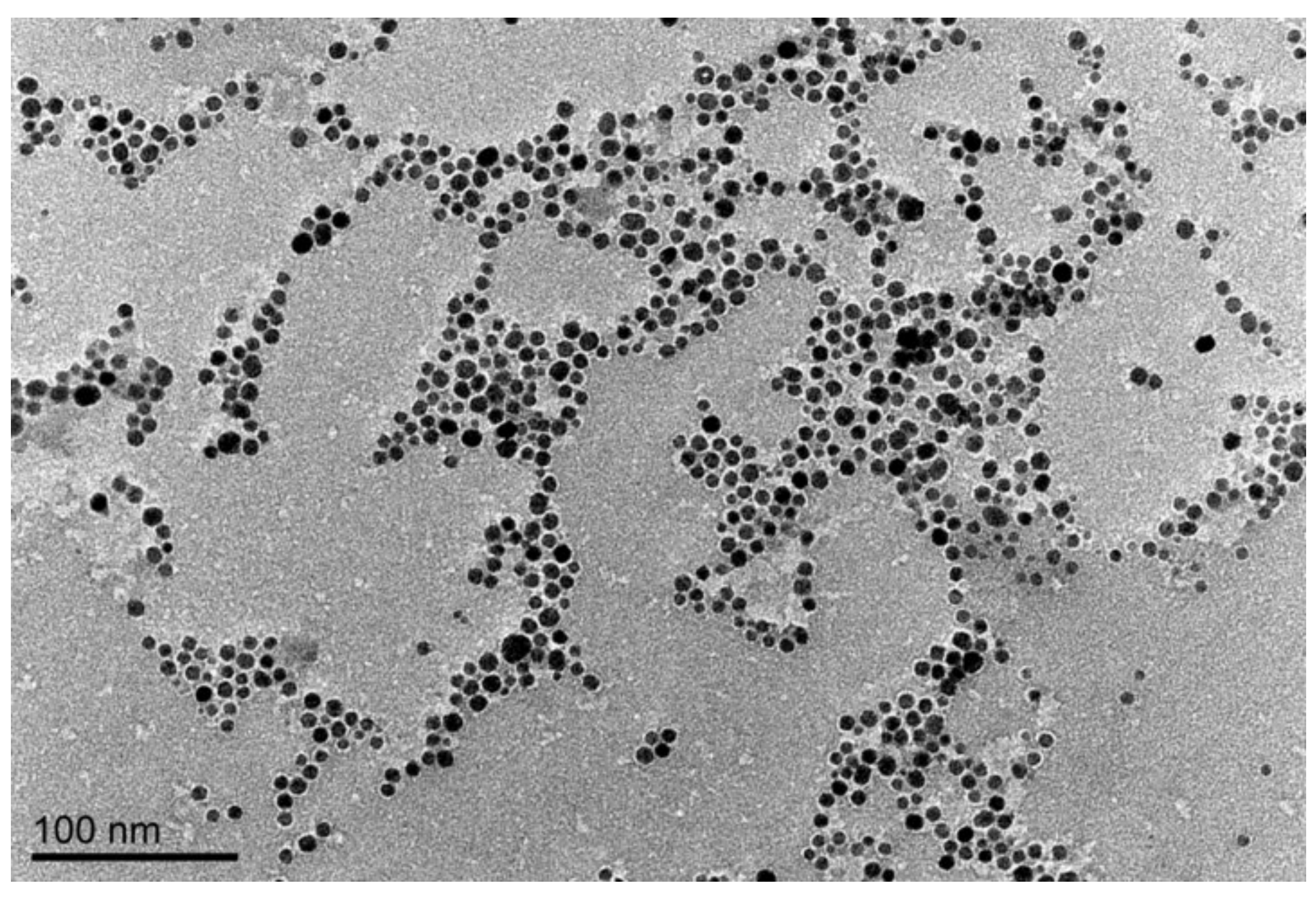
.

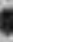

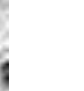

$$
\text { . }
$$

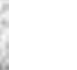

.

$$
\text { . }
$$

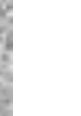
. 30.0000 
Click here to download high resolution image

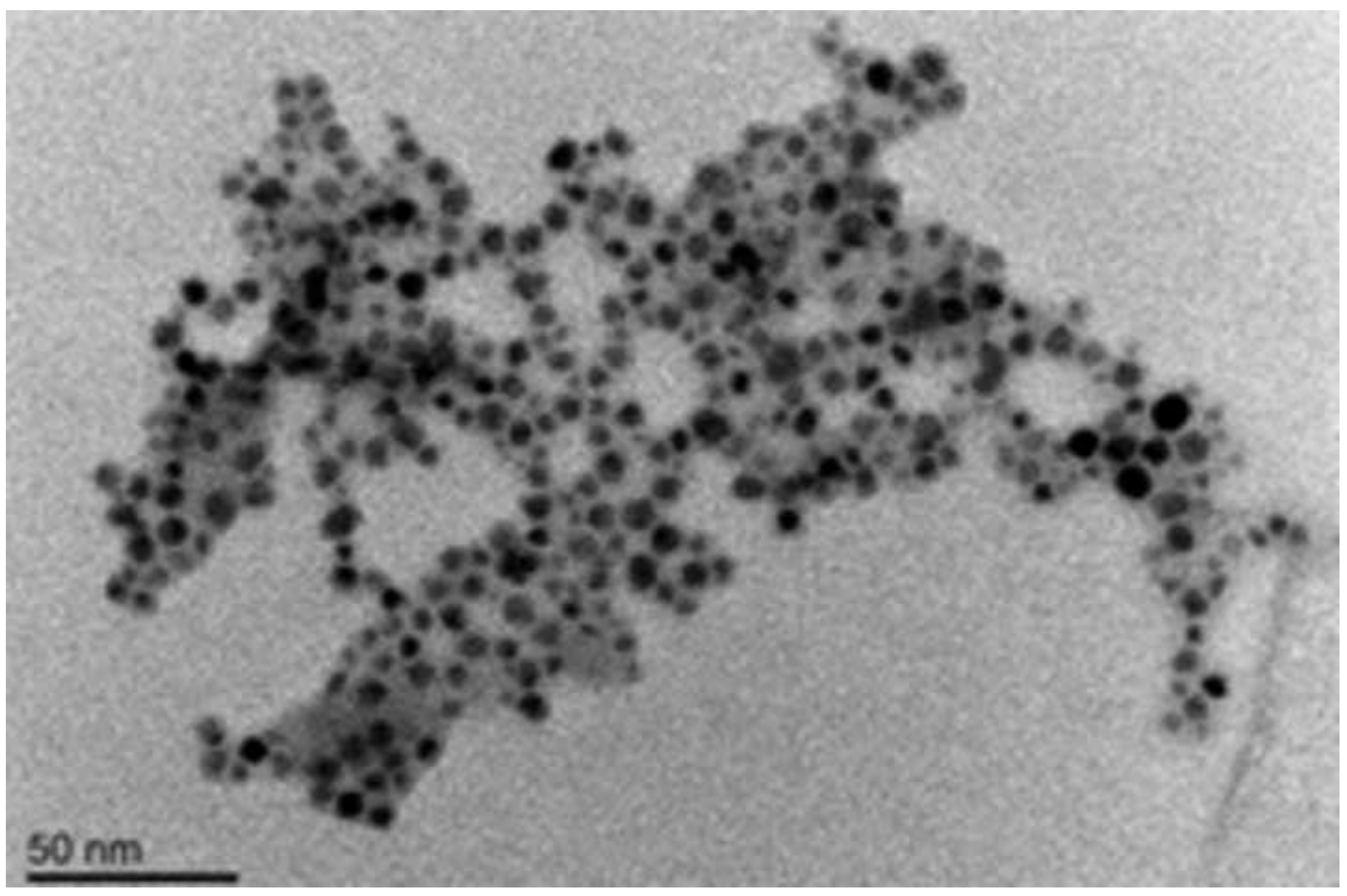

Click here to download high resolution image 
Figure $1 \mathrm{~d}$
Click here to download high resolution image

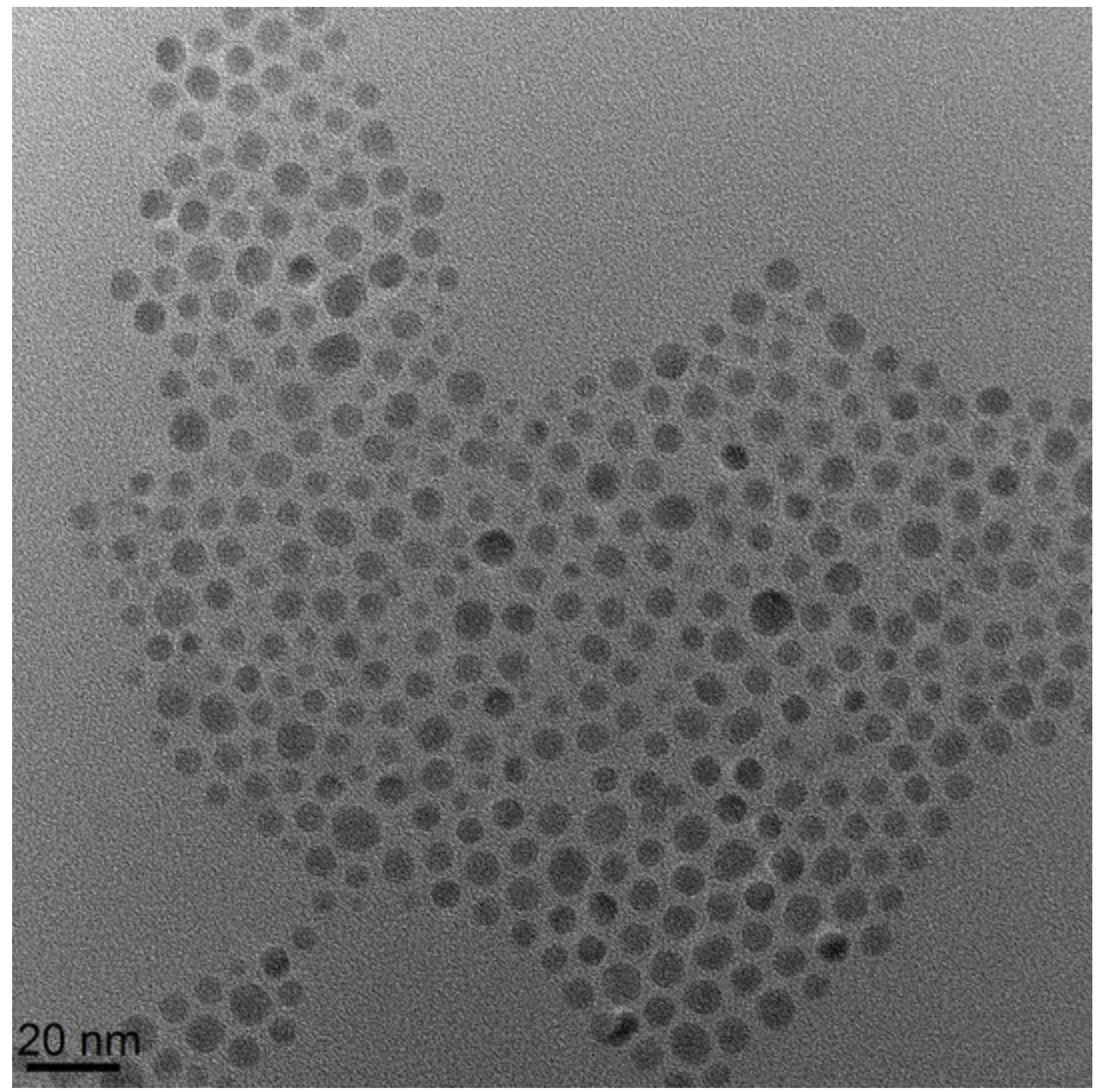




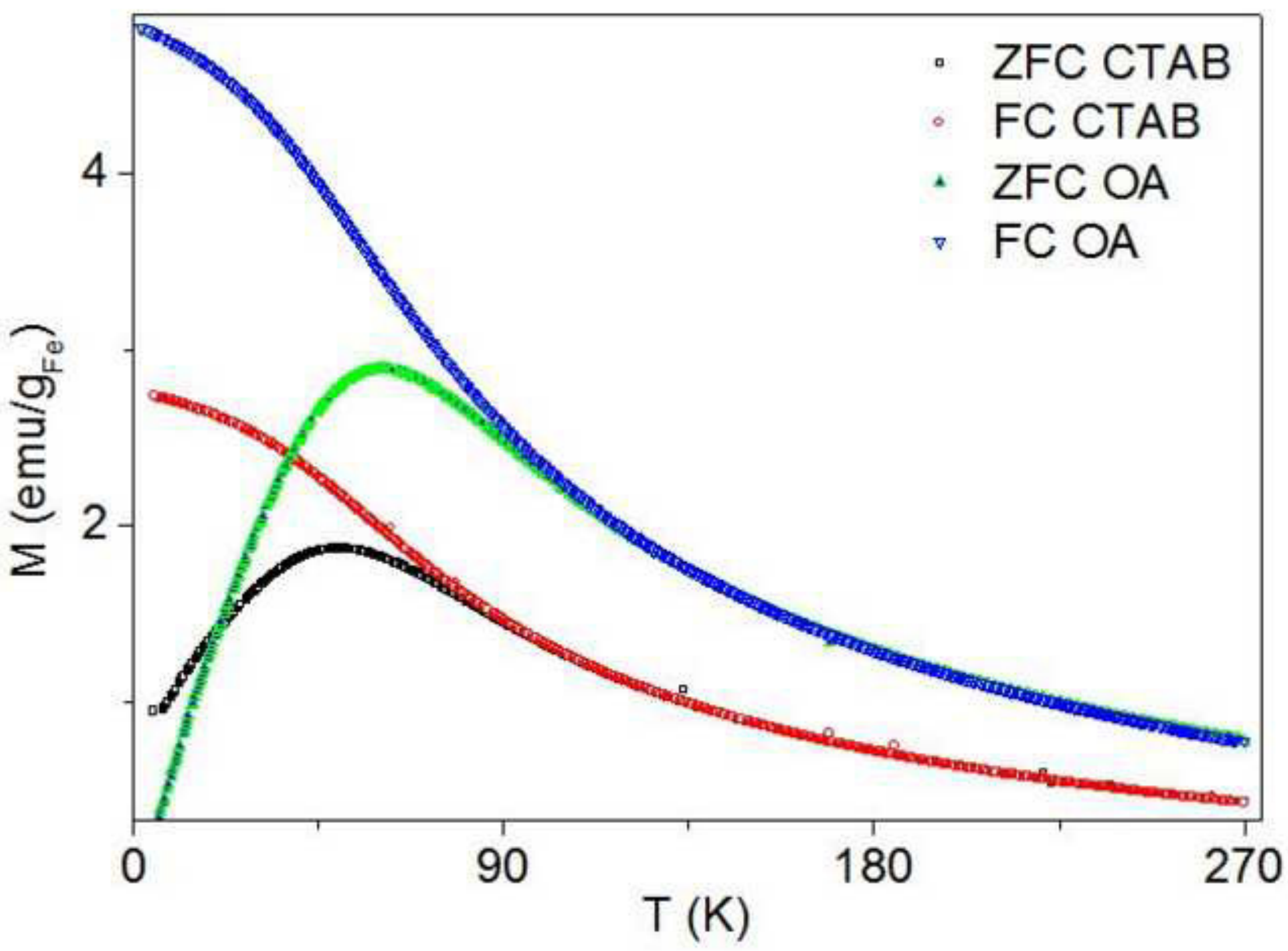




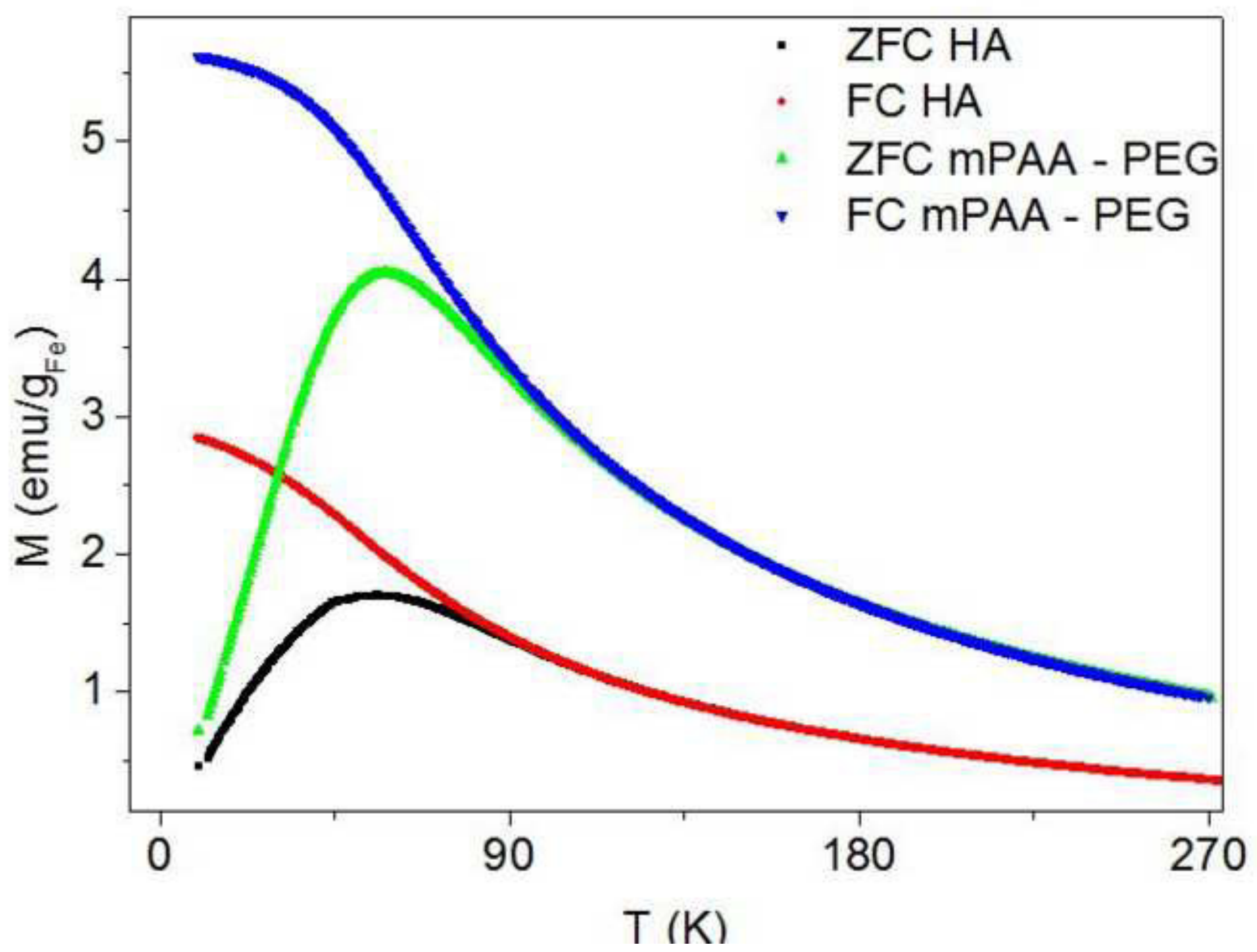




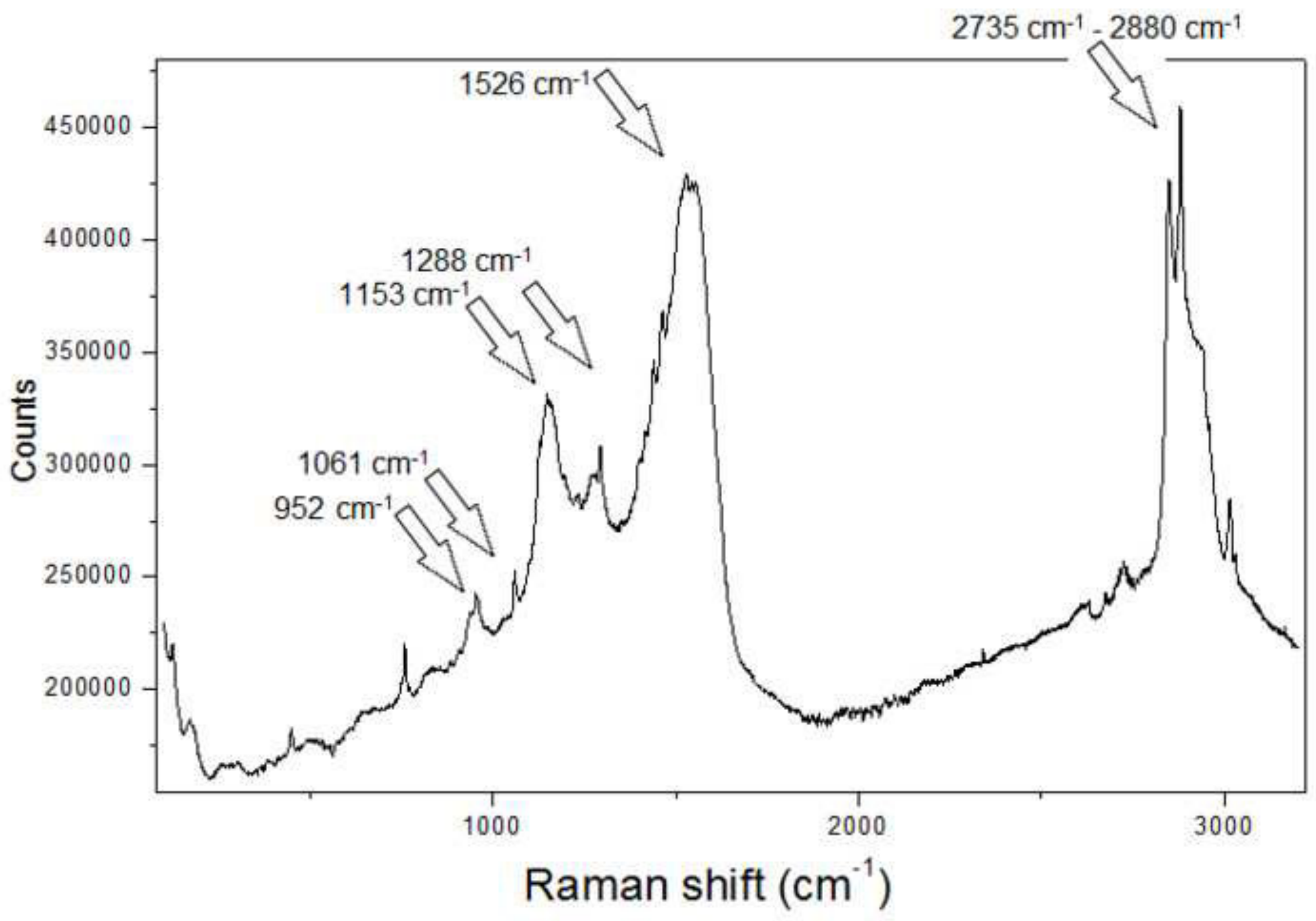




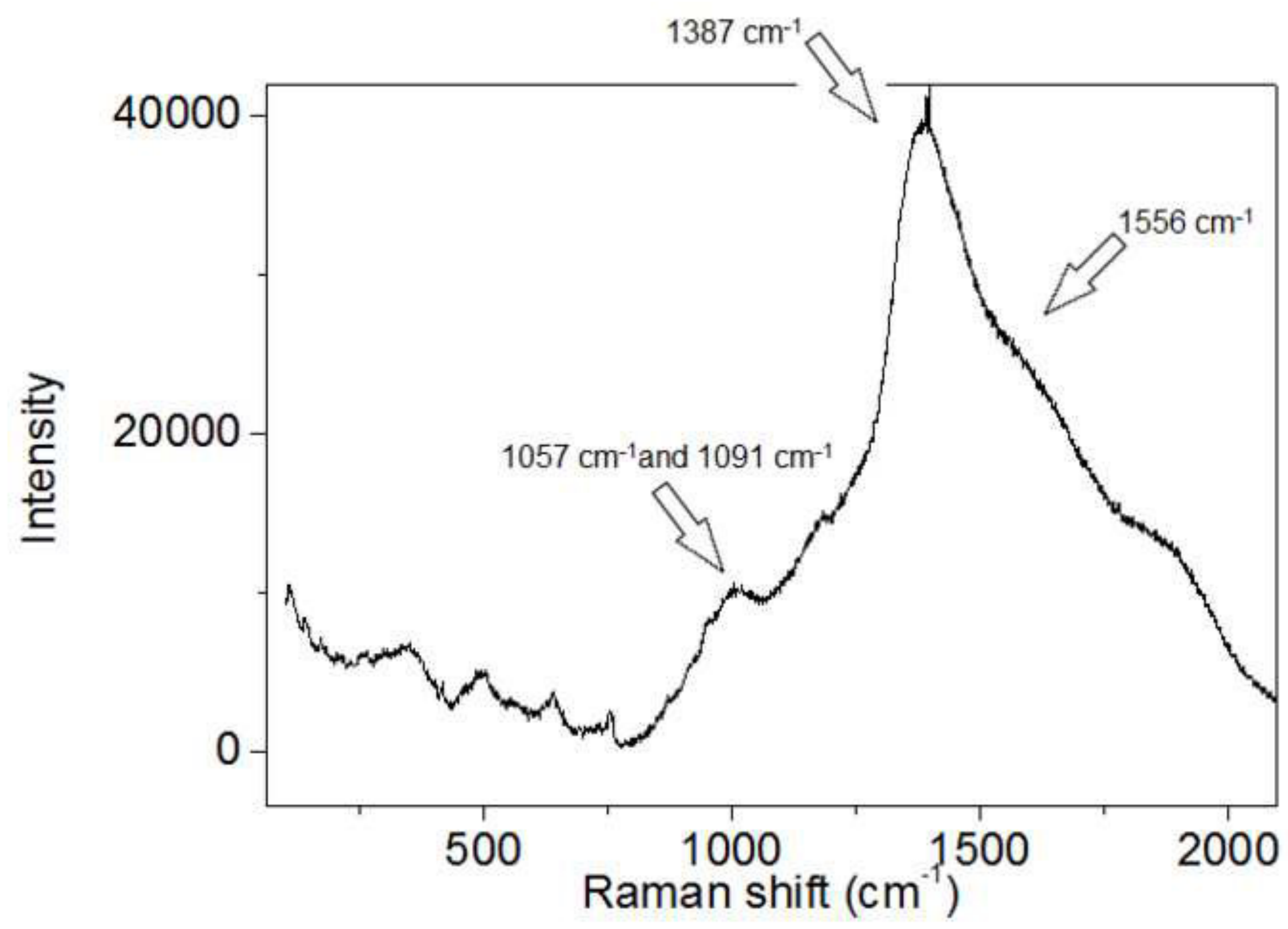




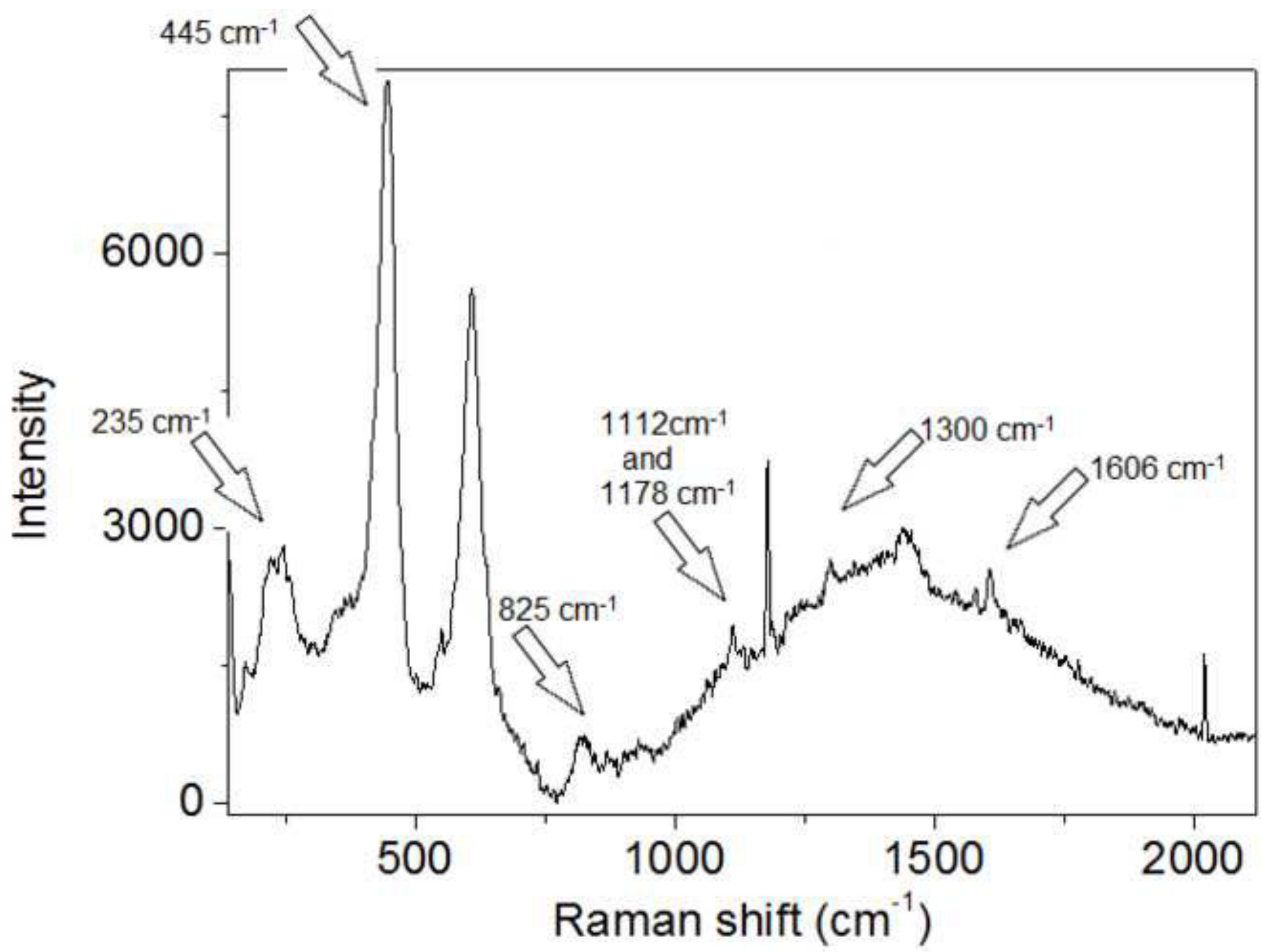




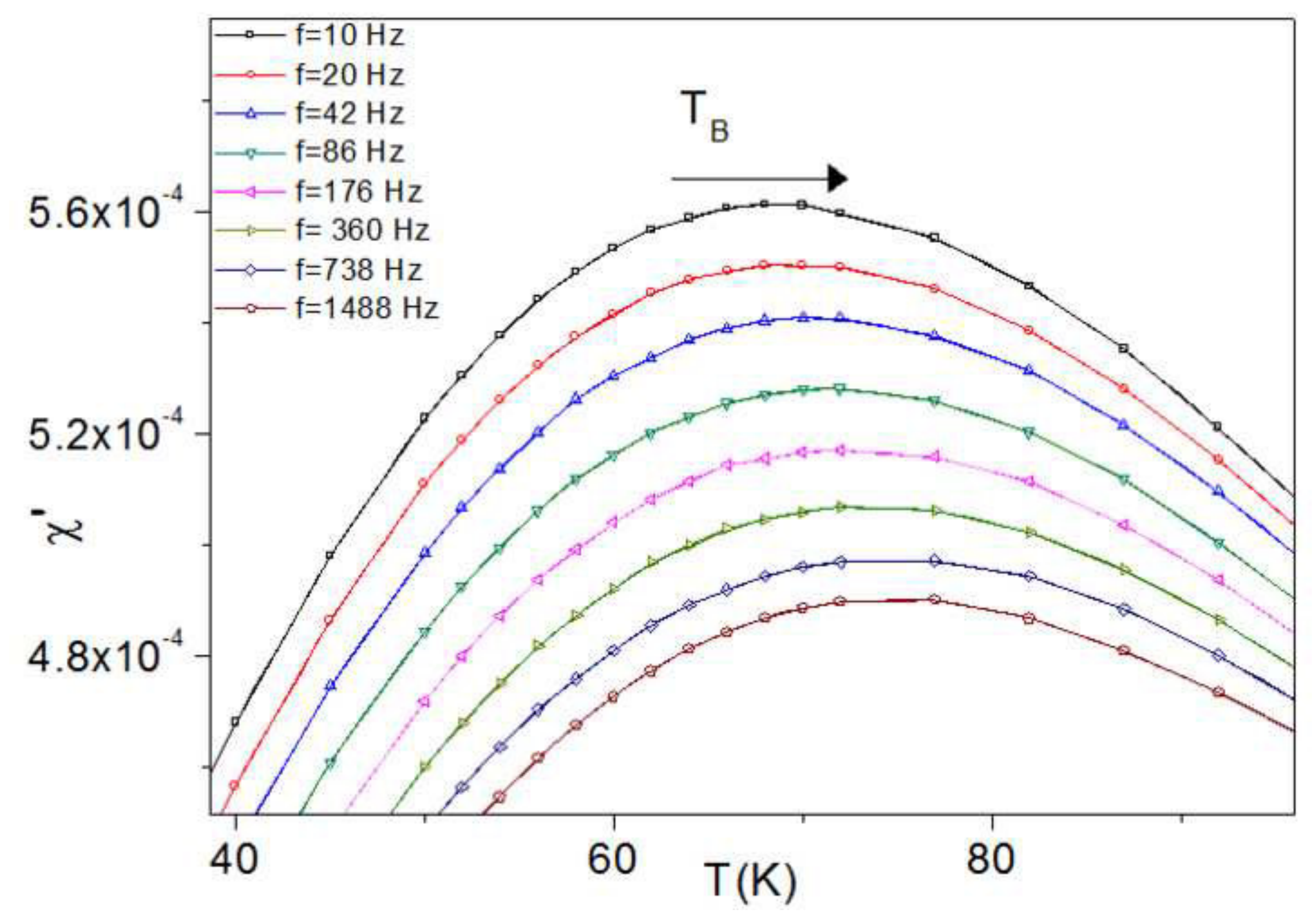




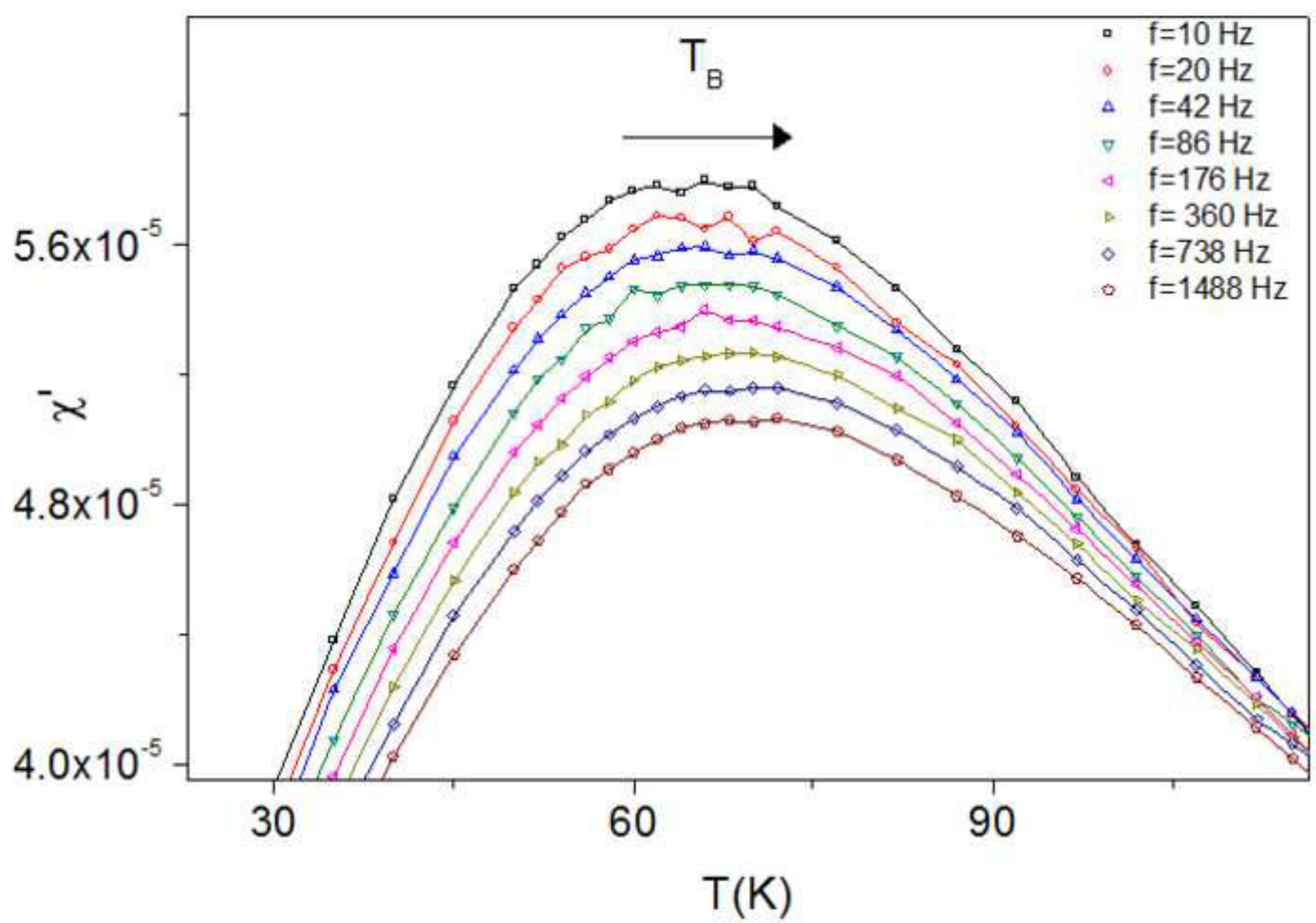




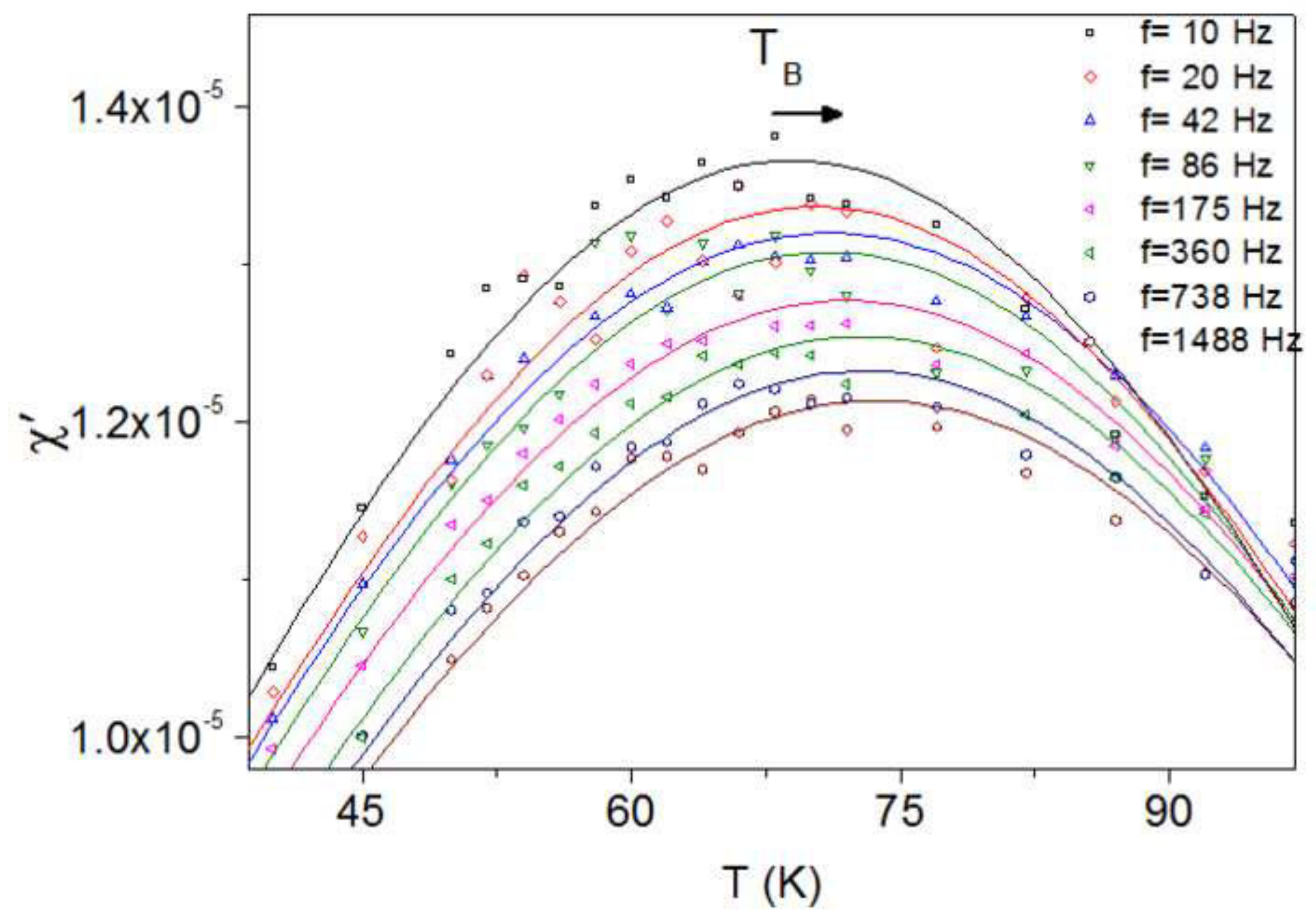




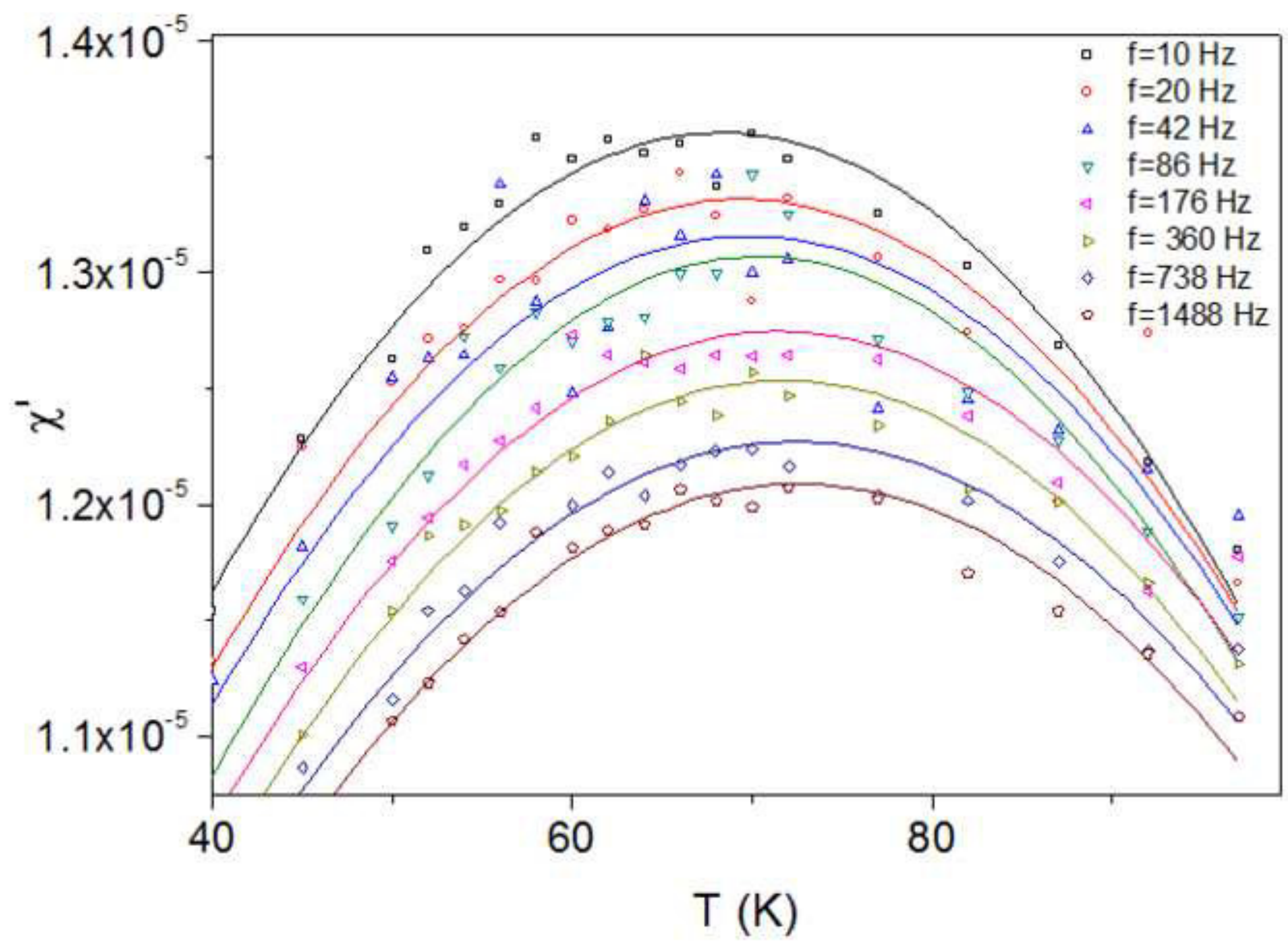


Click here to download high resolution image

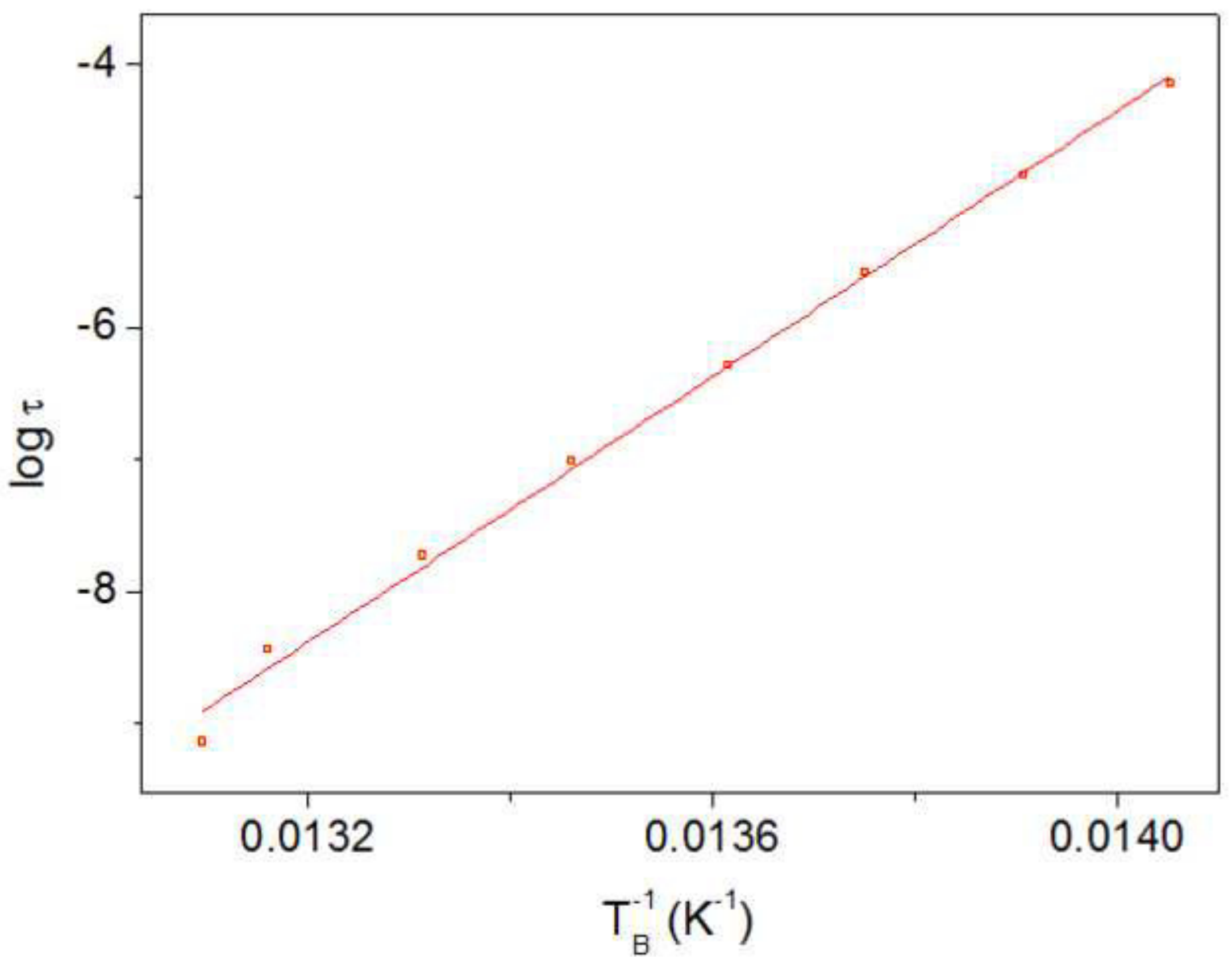




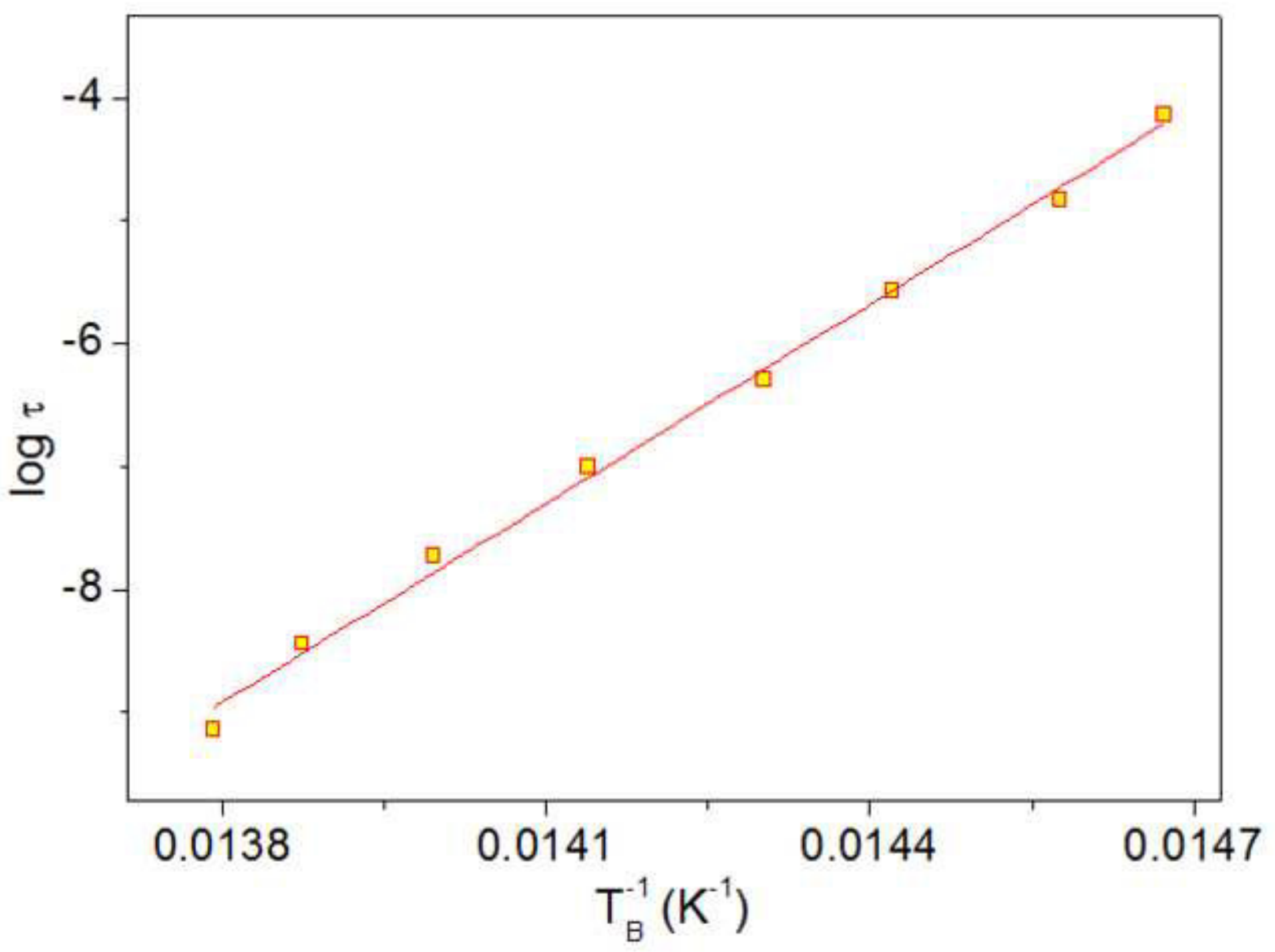


Click here to download high resolution image

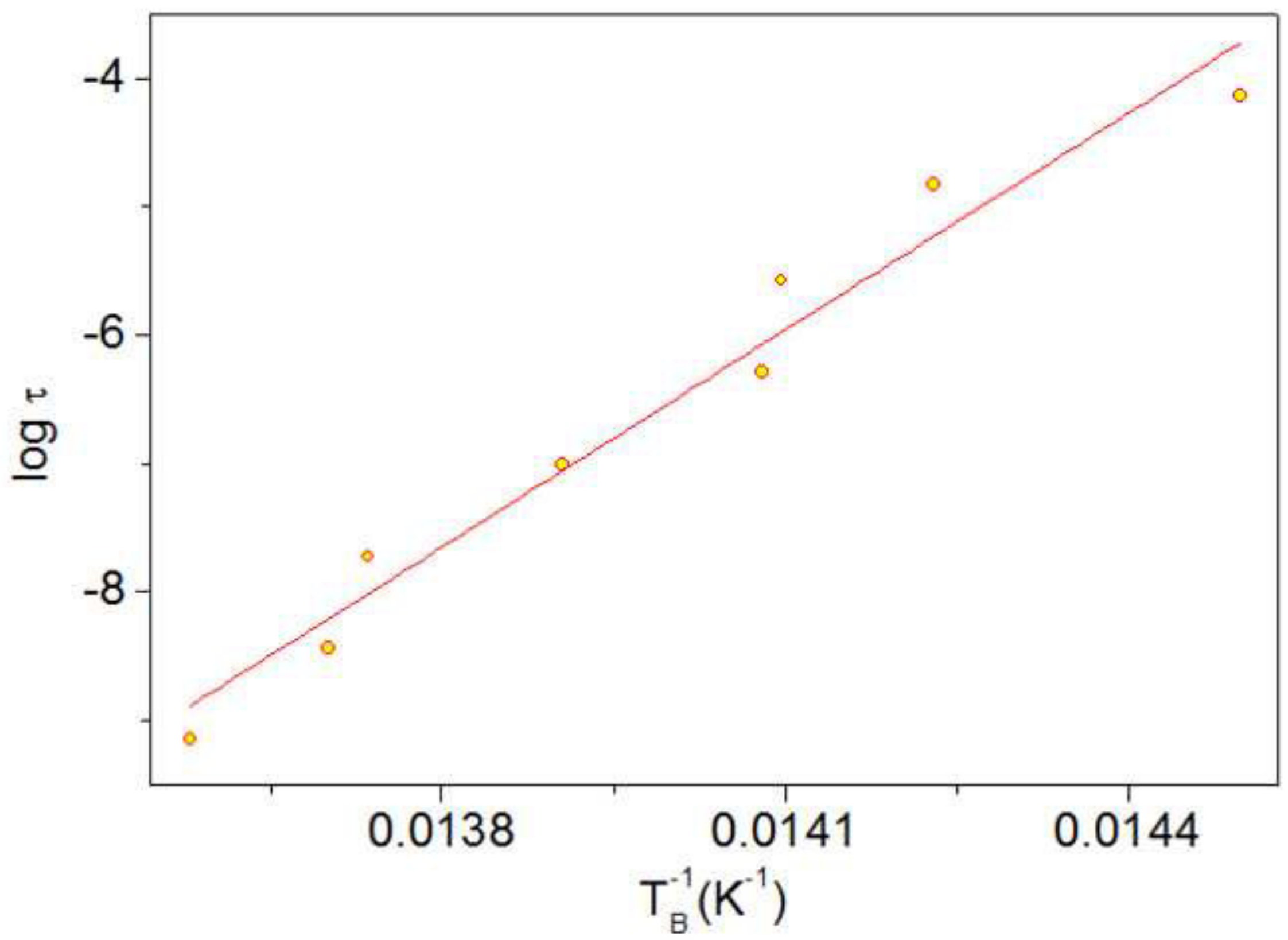




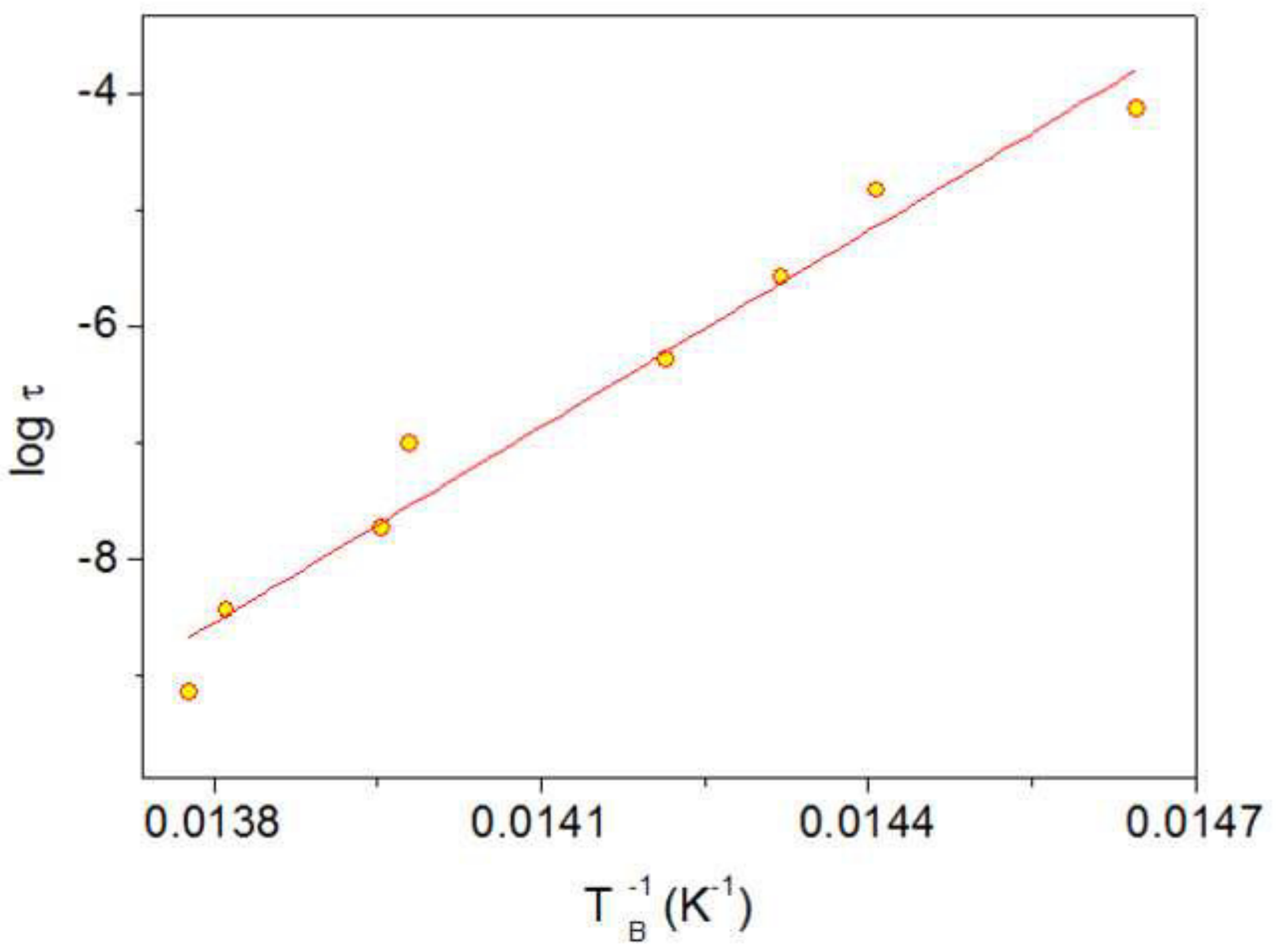




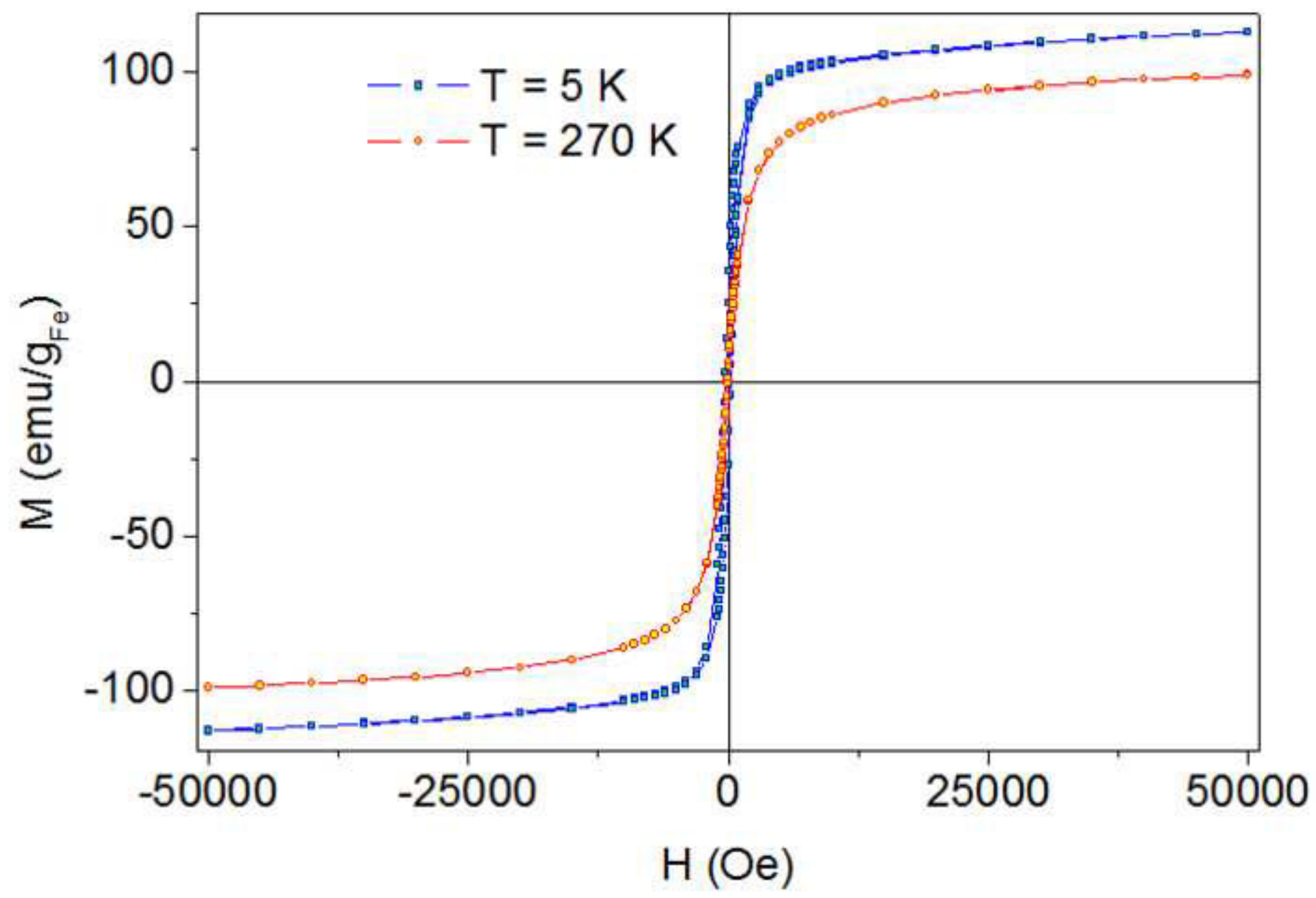




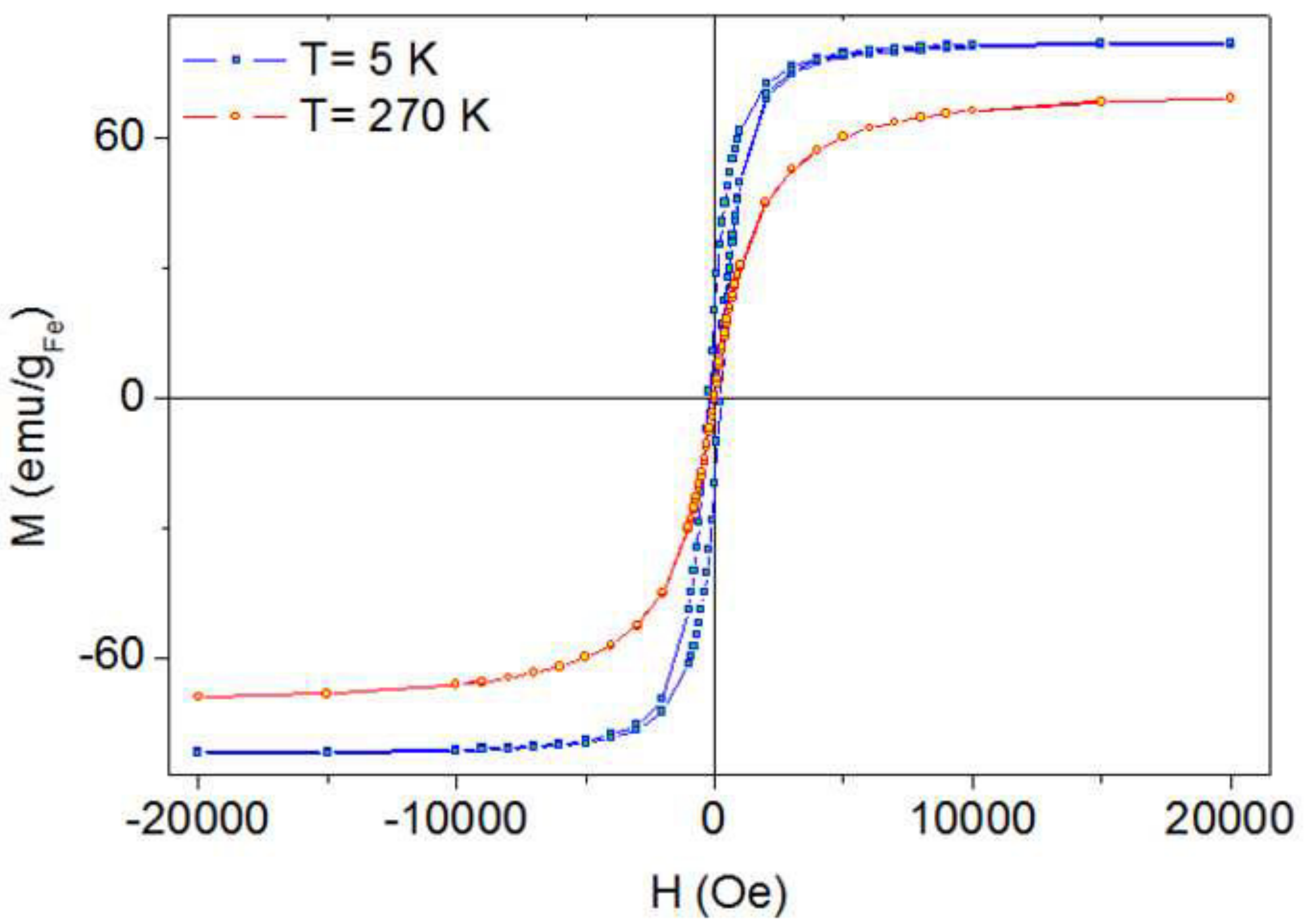




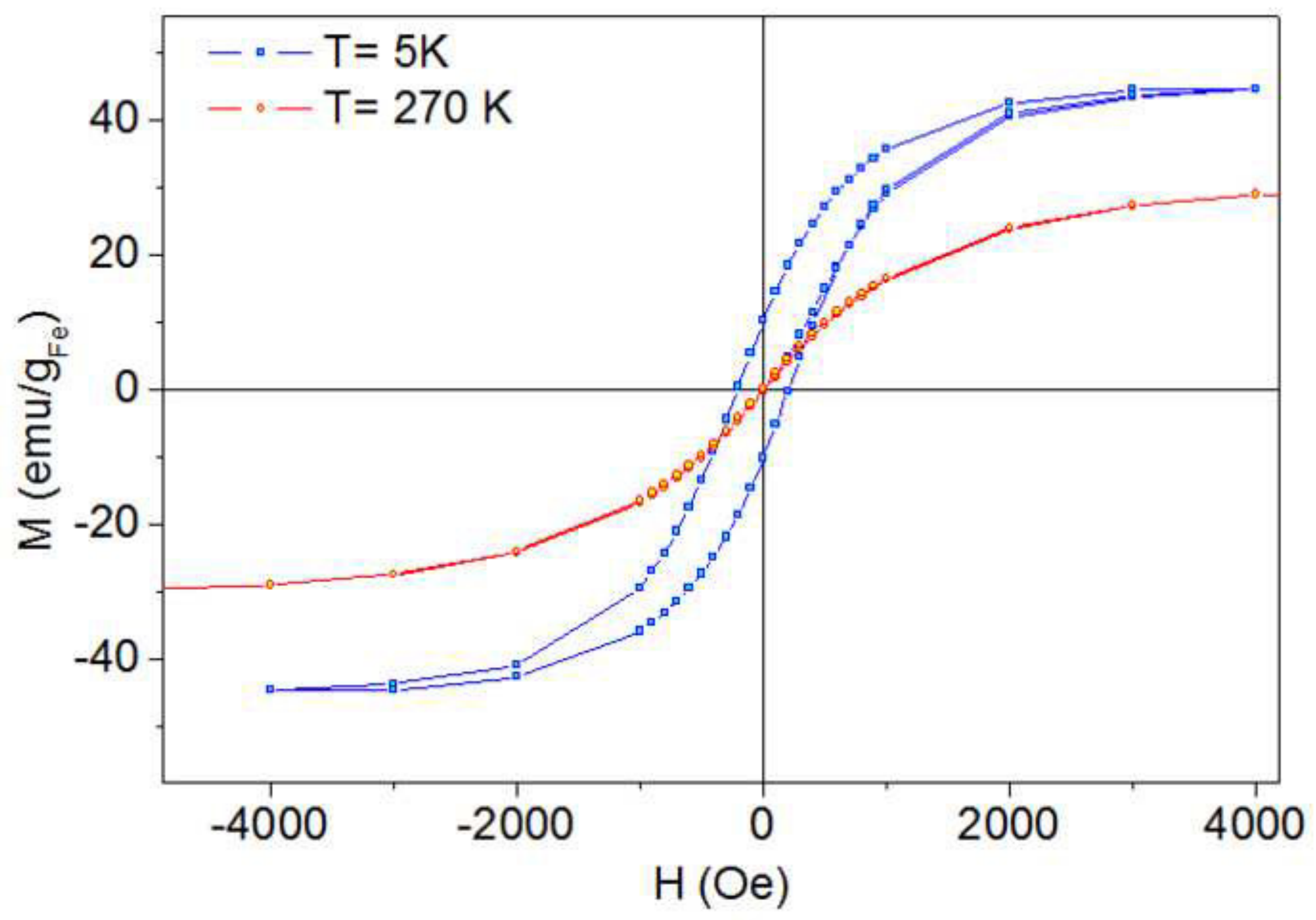




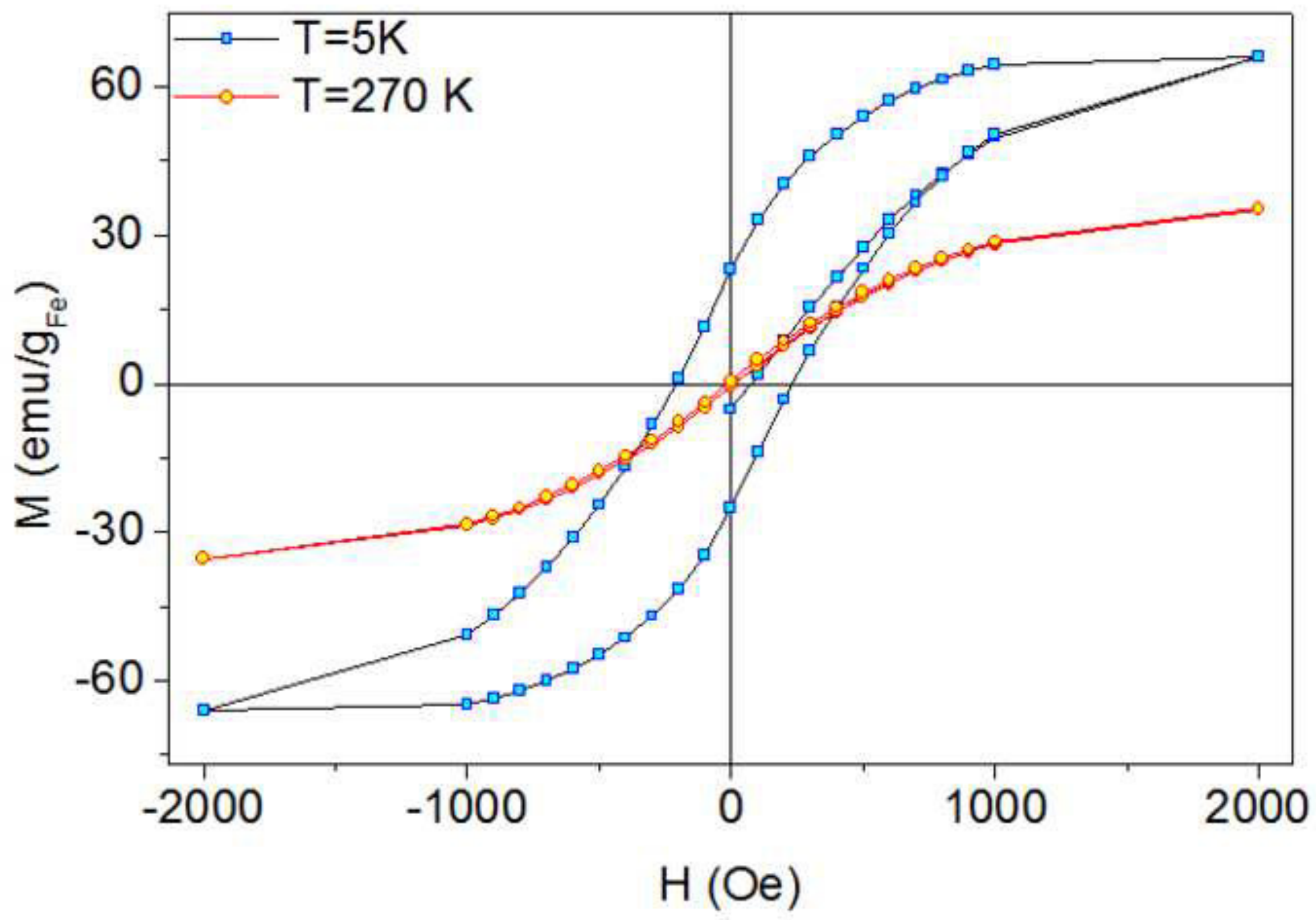


Table 1. Size distribution of nanoparticles

\begin{tabular}{|l|l|}
\hline Number of nanoparticles & Mean size $(\mathrm{nm})$ \\
\hline 341 & 11 \\
\hline 3578 & 12 \\
\hline 3952 & 13 \\
\hline 168 & 14 \\
\hline
\end{tabular}

Table 2. Blocking temperature, Activation energy and effective anisotropy of all four samples

\begin{tabular}{|c|c|c|c|c|}
\hline Sample & $<T_{B}>(K)$ & $\Phi$ & $\begin{array}{c}\text { Activation energy } \\
(\mathrm{K})\end{array}$ & $\begin{array}{c}\text { Effective anisotropy } \cdot 10^{6} \\
\left(\mathrm{erg} / \mathrm{cm}^{3}\right)\end{array}$ \\
\hline Oleic acid & 73,9 & 0,031 & 5040 & 0,60 \\
\hline CTAB & 70,3 & 0,028 & 5398 & 0,65 \\
\hline mPAA-PEG & 71,6 & 0,013 & 5626 & 0,67 \\
\hline HA & 70,8 & 0,012 & 5608 & 0,67 \\
\hline
\end{tabular}

Table 3. Depenedencecoercivity and saturation magnetization of temperature.

\begin{tabular}{|c|c|c|c|c|c|c|c|c|}
\hline \multirow{2}{*}{$\begin{array}{l}\text { Temper } \\
\text { ature } \\
\text { (K) }\end{array}$} & \multicolumn{2}{|c|}{ Oleic acid } & \multicolumn{2}{|l|}{ CTAB } & \multicolumn{2}{|l|}{$\mathrm{HA}$} & \multicolumn{2}{|c|}{ mPAA-PEG } \\
\hline & $\begin{array}{l}\text { Coercivit } \\
\text { y }(\mathrm{Oe})\end{array}$ & $\begin{array}{l}\text { Saturation } \\
\text { magnetiza } \\
\text { tion } \\
\left(\mathrm{emu} / \mathrm{g}_{\mathrm{Fe}}\right)\end{array}$ & $\begin{array}{l}\text { Coerciv } \\
\text { ity }(\mathrm{Oe})\end{array}$ & $\begin{array}{l}\text { Saturation } \\
\text { magnetiza } \\
\text { tion } \\
\left(\mathrm{emu} / \mathrm{g}_{\mathrm{Fe}}\right)\end{array}$ & $\begin{array}{l}\text { Coerciv } \\
\text { ity }(\mathrm{Oe})\end{array}$ & $\begin{array}{l}\text { Saturation } \\
\text { magnetiza } \\
\text { tion } \\
\left(\mathrm{emu} / \mathrm{g}_{\mathrm{Fe}}\right)\end{array}$ & $\begin{array}{l}\text { Coerciv } \\
\text { ity }(\mathrm{Oe})\end{array}$ & $\begin{array}{l}\text { Saturation } \\
\text { magnetiza } \\
\text { tion } \\
\left(\mathrm{emu} / \mathrm{g}_{\mathrm{Fe}}\right)\end{array}$ \\
\hline 5 & 238 & 114 & 214 & 82 & 223 & 66 & 206 & 46 \\
\hline 50 & 38 & 114 & 31 & 80 & 10,5 & 63 & 11 & 41 \\
\hline 100 & 46 & 108 & 15 & 78 & 12,5 & 58,7 & 22 & 38 \\
\hline 200 & 15 & 108 & 16 & 73 & 12 & 45,5 & 12 & 34 \\
\hline 270 & 15 & 102 & 15 & 68 & 12 & 35,4 & 11 & 30 \\
\hline
\end{tabular}

